\title{
Notulæ ad Plantas Japoniæ et Koreæ. IV.
}

\author{
auctore
}

T. Nak a i.

53) Clematis fusca Turcz. v. violacea Maxim. Prim. Fl. Amur. p. 11. Kom. F1. Mansh. II. p. 287.

Korea : Pyeng-yang: 18. VI. 1911 (H. ImaI).

Planta nova ad Floram Koreanam.

FIG. 1.

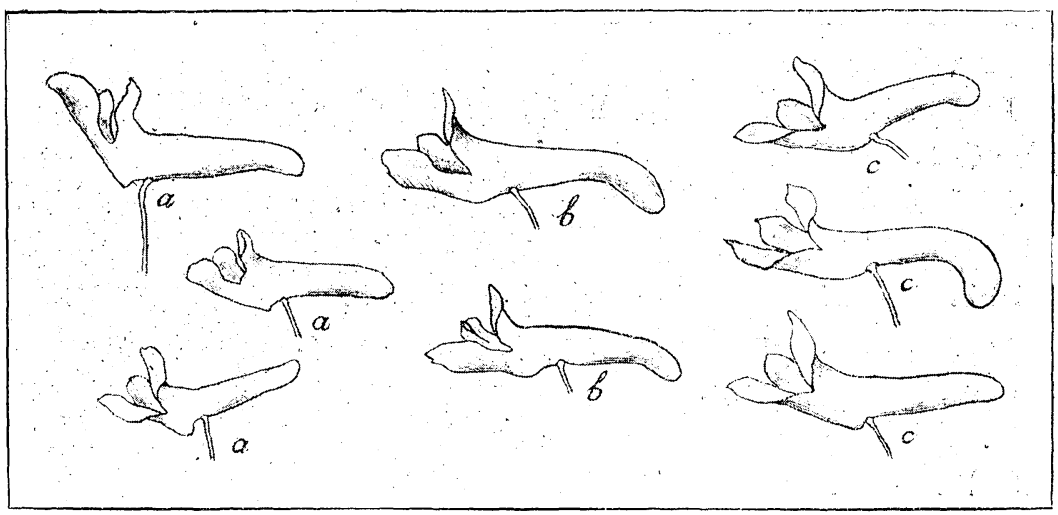

a. Flores Corydalis bulbosæ.

b. Flores C. bulbosæ v. remotæ ex Manshuria et Korea.

c. Flores C. bulbosæ v. remotre ex Japonia.

54) Corydalis bulbosa (Gorter) DC. Fl. Fran. IV. (1805) p. 637.

Fumaria bulbosa GoRTer Flora Ingrica Appendix (1764) p. 201.

F. solida Ehrhart Beiträge Naturk. I. (1787) p. 149.

Corydalis solida (ЕнRH.) Smith Flora Britannica III. (1804) 3. 353.

var. remota (FISCHER). 
Corydalis remota Fischer in Herb. ex Maxim. Prim. Fl. Amur. (1859) p. 37.

Fr. Schmidt Florula Amg.-Burej. p. 33. n. 43. Kom. Fl. Mansh. II. p. 349.

C. bulbosa (non DC.) Turcz. Cat. Baic.-Dah. in Bull. Soc. Nat. Mosc. (1838) n. 98. Franch. Pl. Dav. 28. Forbes et HemsL. in Journ. Linn. Soc. XXIII. p. 36.

C. solida subsp. remota Korsch. in Act. Hort. Petrop. XII. p. 306.

C. gamosepala Maxim. Prim. Fl. Amur. p. 39.

C. ambigua $\beta$. amurensis Maxim. 1. c.

Flores vulgo majores quam in C. bulbosa. Gibber corollæ minus productus. Bracteæ vulgo irregulariter fissæ.

forma 1. genuina Maxim. Prim. F1. Amur. p. 38. Regel Pl. Radd. 139. Kom. Fl. Mansh. II. p. 351.

Folia biternata. Segmenta foliorum elliptica v. rotundata. Tuber vulgo aureo-suffusus.

Lusus. 1. Segmenta foliorum oblongo-cuneata integra v. apice 1-3 fida incisave.

Kobano-engosaku Y. Irnuma Sōmokudzusetsu Vol. XIII. (1856) fol. 4 fig. V.

Hab. Dahuria, China, Manshuria et Korea.

Lusus. 2. Segmenta foliorum oblonga v. elliptica utrinque acuta v. apice obtusa. Tuber vulgo albus.

C. bulbosa a. typica Makino in Tokyo Bot. Mag. VIII. (1894) p. 227.

Engosaku v. Zirobo in Noyama-Sōmokutsūshi I. folio. 33 verso et B. Minamoto Yoshinogunchūbussanshi fol. 14 vers.

Hab. Japonica.

Lusus. 3. Folia triternata. Segmenta foliorum rotundata acuta v. obtusa.

C. bulbosa $\gamma$. rotundiloba Makino in Tokyo Bot. Mag. VIII. (1894) p. 227.

Hab. Nippon.

Lusus. 4. Folia 2-3 ternata. Segmenta foliorum ovatolanceolata, omnia longissime petiolulata.

C. laxa Fran. et Sav. Enum. Pl. Jap. II. p. 372.

Hab. Nippon. 
forma. 2. rotundiloba Maxim. 1. c.

Folia biternata. Segmenta ambitu rotundato-obovata v. rhombeo-cuneata crassiuscula. Tuber vulgo aureo-suffusus.

Lusus. 1. Segmenta foliorum ambitu rotundato-obovata apice truncata $\mathrm{v}$. obtusa integra $\mathrm{v}$. pectinato-incisa.

C. remota v. rotundiloba Maxim. 1. c. Kom. Fl. Mansh. II. 351.

C. solida v. rotundiloba (Maxim.) Regel Pl. Radd. I. p. 139.

C. Vernyi Fran. et Sav. Enum. Pl. Jap. II. p. 273. p. p.

Engosaku in J. Shimada Kwai Herb. II. (1765) p. 10. T. Ono Honzō-Kōmokukeimō Vol. IX. Herb. II. (1803) fol. 14. Zyūtei-honzōkōmokukeimō Vol. IX. Herb. II. (1844) folio 15.

C. ambigua (non Cham. et Schlecht.) Y. Tanaka et $\mathrm{T}$. Ono Yūyōshokubutsudzusetsu (1891) II. n. 404.

Hab. Manshuria et Korea.

Lusus 2. Segmenta foliorum versus apicem lata pectinatoincisa.

C. remota v. pectinata Kom. F1. Mansh. II. p. 350.

Hab. Manshuria.

Lusus 3. Segmenta foliorum versus apicem dilatata, bis tripartita.

C. fumariæfolia Maxim. Prim. Fl. Amur. p. 39.

C. remota v. fumariæfolia (MAxim.) Kom. Fl. Mansh. II. p. 351.

Hab. Manshuria et Korea.

forma 3. lineariloba Maxim. Prim. Fl. Amur. p. 38.

C. lineariloba SiEb. et Zucc. Fl. Jap. Fam. Nat. I. (1843) n. 286.

C. orthoceras S. et Z. 1. c.

C. solida v. linearis Regel Pl. Radd. I. p. 139. MrQ. Prol. F1. Jap. p. 200.

C. solida v. orthoceras MiQ. 1. c.

C. senanensis Fran. et SAv. Enum. Pl. Jap. II. p. 273.

Chikuyo-engosaku S. SATo Sōmoku-rikubu-Kōshuhō. Vol. III. (1874) fol. 20.

Hab. Manshuria, Korea et Japonia. 
forma 4. capillaris Makino in Tokyo Bot. Mag. XII. 119. pro var. Caulis capillaris. Folia 3-4 ternata. Segmenta foliorum parva, rotundato-obovata.

Hab. Shikoku.

forma 5. ternata NAKAI.

Folia ternata. Segmenta foliorum rhombeo-cuneata v. ovata ac margine serrata rarius bisecta.

C. vernyi Fran. et Sav. Enum. Pl. Jap. II. p. 273. p. p.

Ōbano-engosaku in Sōmokudzusetsu Vol. XIII. fol. 4. (1856) fig. IV. Icon. ined. Honzō-zufu Vol. VII. fig. 26 et 27 ? (1839).

Hab. Korea.

55) Triumfetta subpalmata Solander in herb. ex Hemsl. in Journ. Bot. (1890) p. 2. t. 293 I.

T. procumbens (non Forst.) Benth. F1. Hongk. I. p. 273. pro maj. part.

Nippon : insula I woto. XI. 1907. (B. Kawate).

Planta nova ad Floram Japonicam.

56) Euonymus Hamiltoniana WALL, var. Maackii (Rupr.) Kom. F1. Mansh. II. p. 710.

E. Maackii Rupr. in Bull. St. Petersb. XV (1857) p. 358.

C. K. Schneider Illus. Handb. Laubholzk. II. p. 177.

E. Hamiltoniana forma Maackii Maxım. in Mél. Biol. XI. (1881) p. 193.

Korea : monte Chiisan. n. 14. (H. UEKI).

Distr. Manshuria.

57) Euonymus pauciflora Maxim. Prim. Fl. Amur. p. 74. Fr. Schmidt. Fl. Amg.-Burej. n. 90. Kom. Fl. Mansh. II. p. 705. C. K. Schneider Illus. Handb. Laubholzk. II. p. 170 fig. 112. $\mathrm{g}-\mathrm{h}$.

E. verrucosa var. pauciflora (Maxim.) Regel Tent. Fl. Uss. n. 120. Maxim. in Mél. Biol. XI. p. 195. Korsch. in Act. Hort. Petrop. XII. p. 320.

Korea: monte Chiisan n. 22. (H. UEKI).

Distr. Regio Ussuriensis et Manshuriensis.

Planta nova ad Floram Koreanam. 
58) Koelreuteria paniculata LaxM. Nov. Comm. Petr. XVI. p. 561. t. 18. MiQ. Prol. Fl. Jap. p. 256. Fran. et Sav. Enum. Pl. Jap. I. p. 85. DC. Prodr. I. p. 616. Franch. Pl. Dav. p. 75. Forbes et Hemsl. in Journ. Linn. Soc. XXIII. p. 138.

Nom. Jap. Muku-genzi.

Korea: Mulgeum. 11. X. 1902. (T. Uchiyama).

Distr. China.

59) Raphiolepis umbellata (Thunb.) Makino in Tokyo Bot. Mag. XVI. (1902). p. 13. C. K. Schneider Illus. Handb. Laubholzk. I. (1906) p. 705 fig. $390 \mathrm{~h}-\mathrm{i}$ et in FEDDE Repert. (1907) p. 152.

Laurus umbellata Thuni. Fl. Jap. (1784) p. 175.

Raphiolepsis japonica Sieb. et Zucc. F1. Jap. I. (1835) p. 162. t. 85. Fran. et Sav. Enum. P1. Jap. I. p. 142.

Nom. Jap. Sharinbai.

Korea : Chölla : Mokpho 1911. (H. UEKI).

60) Glossogyne tenuifolia Cass. DC. Prodr. V. p. 632. Benth. Fl. Hongk. p. 184. et F1. Austr. III. p. 544. Seem. F1. Vitiensis p. 144. Forbes et Hemsl. in Journ. Linn. Soc. XXIII. p. 436. Matsum. et Hayata Enum. Pl. Form. p. 206.

Nom. Jap. Seriba-Sendangusa.

Nippon: insula Iwōtō. XI. 1907. (B.Kawate).

Planta nova ad Japoniam propriam. p. 437 .

61) Ligustrum medium Fran. et Sav. Enum. Pl. Jap. II. Nom. Jap. Oba-ibota.

Korea: monte Chiisan 1911. (H. UeKr)

Planta nova ad Floram Koreanam.

62) Gentiana scabra Bunge $\alpha$. Bungeana Kusnez. forma angustifolia Kusnez. NakaI F1. Kor. II. p. 99.

Korea : circa Suigen 1911. (H. Uekr).

63) Mazus stachydifolius (Turcz.) Maxım. in Mél. Biol. IX. p. 404. Franch. Pl. Dav. p. 222. Forbes et HemsL. in Journ. Linn. Soc. XXVI. p. 183. Freyn. in Oest. Bot. Zeit. (1902). p. 402. Kom. Fl. Mansh. III. p. 418. 
Tittmannia stachydifolia Turcz. Bull. des Natur. de Mosc. XI. p. 156.

Vandellia stachydifolia (Turcz.) WaLP. Repert. III. p. 214.

V. oborata Herder in Act. Hort. Petrop. I. p. 580.

Korea : circa Pyeng-yang. 18. VI. 1911. (H. IMAI).

Planta nova ad Floram Koreanam.

64) Phyllanthus (Euphyllanthus) boninsimæ NAKaI sp. nov. Perennis? Planta basi lignosa ramosa. Rami hornotini filiformes flexuosi. Stipula ovato-lanceolata acutissima $1 \mathrm{~mm}$. longa integerrima v. obsolete serrulata. Folia disticha elliptica v. oblongo-elliptica utrinque acuta $\mathrm{v}$. obtusiuscula penninervia, subtus pallidiora 2-12 mm. longa, 1-4 mm. lata. Flos $\hat{s}$ sepalis 6, obovatis, apice obtusissimis; glandulis 6 obovato-orbiculatis, sepalis alternis; staminibus 3 toto connatis; antheris horizontali dehiscentibus. Flos + p sepalis 5, obovato-lanceolatis apice acutinsculis; stylis 3 patentibus bifidisve. Capsula globosa laevia. Semina longitudinali striati simulque transverserugosi.

Bonin : Omura IV. 1906. (B. Kawate).

$\mathrm{Ph}$. Niruri affinis sed sepalis 6 et forma styli, foliis acerioribus ac laxius dispositis differt.

65) Triglochin maritima L. Sp. Pl. p. 483. Maxim. Prim. Fl. Amur. p. 267. Mig. Prol. Fl. Jap. p. 71. Fran. et Sav. Enum. P1. Jap. II. p. 17. Aschers. et Graebn. Mitteleuropafl. I. p. 376. Thомe Fl. Deutsch. Oest. Schw. I. p. 79. Kom. Fl. Mansh. I. p. 229. Buchenau Schuechzeriaceæ p. 8.

T. atacamensis PhIL. Fl. Atac. (1860) p. 49. p. p.

Nom. Jap. Shibana.

Korea: Chemulpo, 17. IX. 1902 (T. Uchiyama).

Distr. in litore maris bor. hemisphaericæ.

Planta nova ad Floram Koreanam.

66) Eriocaulon cauliferum Makino in Tokyo Bot. Mag. XXIV. p. 165 cum. fig.

Korea : circa Suifien no. 57 (H. UERI). 
Speciei E. bifistuloso quum non vidi arcte affinis esse videtur; Ex descriptionibus exquo tantum differt. Caule semper simplici. Petalorum lobis florum masculorum apice ciliatis atque nigropunctatis. An varietas propria?

Planta nova ad Floram Koreanam.

67) Platanthera Okuboi Makino in Tokyo Bot. Mag. XIX. p. 25.

Bonin : Kiyose III. 1906 (B. KaWATE).

Planta nova ad Floram Boninensem.

68) Schœnus (Paniculatæ) Hattorianus NaKaI sp. nov. Rhizoma repense squamis brunneis imbricatis obtectum. Caulis proxime congestus $2-6$ pedalis v. ultra teres. Folia 1-2, omnia basilaria vaginantia, ore barbata 1-2 pollicaria. Lamina foliorum rigida lanceolata acutissima $5-8 \mathrm{~mm}$. longa. Bracteæ vaginantes $4-7 \mathrm{~mm}$. longæ, laminis $4-5 \mathrm{~mm}$. longis acutis continuæ, vaginis ore barbulatis. Panicula secunda laxa. Spicula longe-pedunculata, pedunculis gracilibus semiteretibus v. obsolete-triangularibus. Squamæ involucrantes distichæ utrinque 3-4, interiores longissimæ 6-7 mm. longæ, extima brevissima $1 \mathrm{~mm}$. longa late-ovata ; onmes fuscæ. Glumæ quisque flore unicæ lanceolatæ fuscæ. Flores hermaphroditi. Stamina tria. Antheræ lineares. Styli parum exerti apice trifidi sed conniventes. Ovarium obovoideum triangulare, faciebus reticulatis, angulis elevatis.

Nom. Jap. Zyoi ie. Juncus 10 pedalis.

Bonin: Hatsune (H. Hattori et B. Kawate).

Species Anstraliensis et Borneo-philippinensis S. melanostachyus proxima venit sed laminis foliorum expansis, bracteis vaginantibus lanatis, glumis florum obtusis, seminibus tuberculatis $a b$ hac speciei distat.

69) Ischæmum australe R. BR. Prodr. p. 205. Benth. Fl. Austr. VII. p. 519.

Andropogon cryptatherus Stend. Syn. Glum. I. p. 376.

Bonin : insula Minamishima XII. 1906 (B. Kawate).

Planta nova ad Floram Japonicam. 
70) Panicum flavidum Retze. Obs. IV. p. 15. Kunth Enum. I. p. 78. Benth. Fl. Austr. VII. p. 434 Hook. fil. Fl. Brit. Ind. VII. p. 28.

P. brizoides Trin. Gram. t. 158.

Bonin. XII. 1910. (B. KaWATE).

var. distans (Trin.) Hook. fil. 1. c. p. 29.

P. distans Trin. Gram. t. 172.

Bonin. XII. 1910 (B. Kawate).

Distr. sp. et var. Asia austr. et Australia.

Plantae novae ad Floram Japonicam.

\section{Artemisiæ Japonicæ.}

Sect. I. Dracunculus Bess. in Bull. Soc. Nat. Mosc. VIIII. (1835) p. 1. DC. Prodr. VI. (1837) p. 93. Maxim. in Mél. Biol. VIII. p. 523.

Flores radii feminæi fertiles, disci bisexuales sed abortu ovarii steriles.

(Radix annua, Folia caulina bipinnata glabra, lacinis capillaribus, $5 \mathrm{~mm}$. latis. Inflorescentia paniculata. Capitula

1. nutantia minima $1 \mathrm{~mm}$. vix lata. Involucri squamæ glaberrimæ....................................Art. scoporia WALDST. et KIT.

Radix perennis. 2.

Capitula obconica basi turbinata apice $3 \mathrm{~mm}$. lata. Folia radicalia longissime-petiolata ambitu rhomboidalia bipinnata, lacinis angustis $1.5 \mathrm{~mm}$. latis. Folia caulina pinnata. 2. \{nflorescentia paniculata. Capitula nutantia. .................................................... Art. Fukudo Makino.

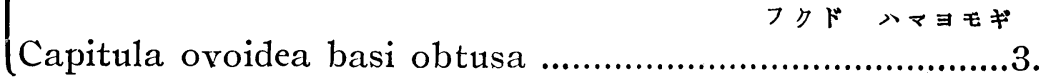

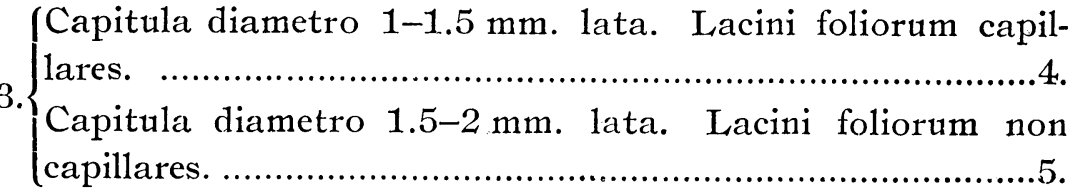


4.

(Folia caulina glaberrima v. glabrescentia, radicalia sericea.

Art. capillaris ThunB. カハラョモギ

Folia caulina sericea, radicalia subargenteo-sericea.

Art. capillaris v. sericea NaKaI.

(Caulis et inflorescentia juvenilis fusco-lanatus. Folia bipinnatifida v. secta, lacinis lanceolatis v. linearibus. Capi5. tula globosa Art. desertorum SPRENG. Caulis et inflorescentia juvenilis glaberrimus v. pilosus. Capitula ovata 6.

Folia simplicia apice varie incisa. Art. japonica ThunB. Folia caulina cuneata. Inflorescentia densa. forma typica NAKAI. キトコヨモギ

Folia caulina obovata, basi subito attenuata. Inflorescentia 6. \{dense-congesta forma spatulata NakAI. Folia ut in typica sed flores laxissime dispositi. forma laxiflora NakAI.

Folia 1-2 pinnatifida, lacinis linearibus. forma resedifolia TAKEDA. ホソバキトコョモギ

71) Artemisia capillaris Thunb. Fl. Jap. p. 310. var. sericea NAKAI

Folia radicalia bipinnata, segmentis linearibus subargenteosericeis ; caulina pinnata, segmentis capillaribus sericeis. Inflorescentia racemoso-paniculata ambitu semifusiformis, rhachibus et pedicellis sericeis. Capitula ut in typo.

Nippon: Hachinohe VIII. 1900 (N. Kinashi) prov. Rikuchu (Y. Chiba).

72) Artemisia japonica Thunb. Fl. Jap. p. 310.

forma spatulata NAKAI.

Folia caulina obovata basi subito-attenuata. Infloresentia dense congesta.

Nippon: circa Mito X. 1911. no. 54 (I. Ando). 
forma laxiflora NAKAI.

Folia caulina cuneata apice varie incisa. Inflorescentia laxissima.

Nippon: circa Mito X. 1911 no 50 (I. Ando).

Sect. II. Abrotanum Bess. in Nouv. Mém. Soc. Nat. Mosc. III. p. 105. DC. Prodr. VI. p. 105.

Capitula hetetogama. Flores radii foeminei, disci hermaphrodites, omnes ferties.

1. $\left\{\begin{array}{l}\text { Radix annua } \\ \text { Radix perennis }\end{array}\right.$ 2.

(Folia tripinnatisecta glabra, lacinis angustis $0.5-1 \mathrm{~mm}$. latis.

Capitula parva $1 \mathrm{~mm}$. lata nutantia..............Art. annua $\mathrm{L}$.

2. Folia 2-3 pinnata glabra, lacinis linearibus $1 \mathrm{~mm}$. latis. Inflorescentia paniculata. Capitula nutantia 3-4 mm. lata. Art. apiacea Hance. カハシニンジン

3 Flores vulgo diametro $3 \mathrm{~mm}$. excedentes. .........................4.

Flores diametro $3 \mathrm{~mm}$. vix attingentes. ..........................8.

Inflorescentia corymbosa. Folia caulina pinnatifida, in-

4. volucri squamæ extus albidæ............Art. glomerata LEDEB.

Racemus simplex v. paniculatus.......................................5.

Involucri squamæ glaberrimæ. Folia cuneata apice varie incisa v. serrata, utrinque sparse arachnoidea. Flores longis-

5. simepedunculati nutantes diametro 5-10 $\mathrm{mm}$.

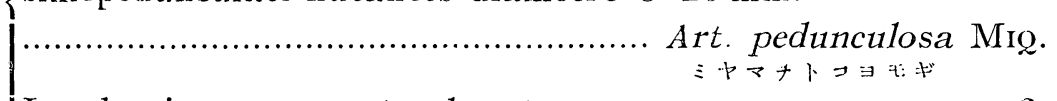

Involucri squamæ extus lanatæ ....................................6.

(Folia simplicia acute-dentata supra sparse arachnoidea, dentibus elongato-triangulatis v. lanceolata-triangulatis. 6. Flores paniculati vulgo erecti 3-4.5 mm. lati. 5-6 longi.

Folia pinnatifida utrinque lanata v. supra dense arachnoidea.

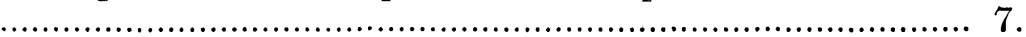

(Racemus spicatus. Flores diametro 6-12 mm. lati, hemis7. phærici Art. stelleriana Bess.

Racemus paniculatus. Flores diametro $3-4.5 \mathrm{~mm}$. lati. Art. stelleriana Bess. v. sachalinensis NAKAI. 
(Folia tripinnata supra viridia, subtus pallidiora glabra v. 8. pilosa. Inflorescentia paniculata. Flores nutantes. ......9. Folia simplicia v. tantum pinnta et si bipinnatisecta subtus incana..... 10 .

(Rachis foliorum integra. Lacini foliorum lanceolati. Racemus paniculatus. Capitula globosa nutantia 2-3 mm. lata. ..Art. laciniata Wil.d. v. laciniata Maxim. 9. Rachis foliorum pectinata...................... Art. sacrorum LEDEB. (Lacini foliorum $1 \mathrm{~mm}$. lati elongati. Folia 5-6 cm. lata. ... ..v. laciniæformis NaKaI. Lacini foliorum $1.5-2.5 \mathrm{~mm}$. lati. Folia $4-5 \mathrm{~cm}$. lata, subtus pallidiora.

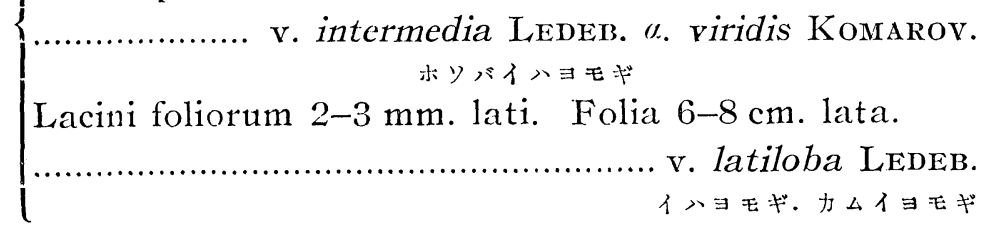

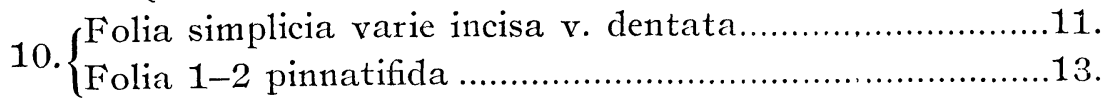

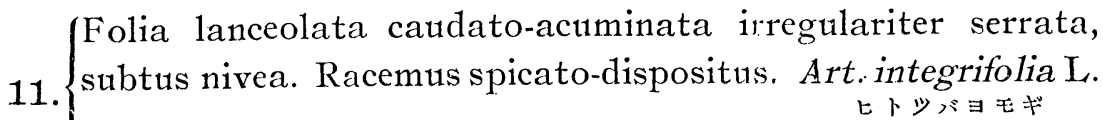

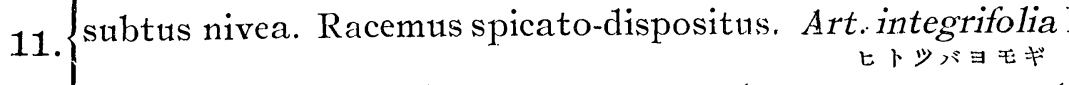

Folia obovata v. subspatulata grosse-dentata.................12.

Capitula ovata incana v. dense arachnoidea. Folia subtus v. utrinque incana, lacinis stipulaceis vulgo evolutis. stolonifera............................Art. stolonifera (Maxim.) Kom.

12. Capitula globosa glaberrima. Planta a gemmis v. a sobolibus, v. a rhizomatibus ascresens. ........Art. Keiskeana MiQ. イメョれギ

Folia utrinque glabra ...............forma $\alpha$. typica NaKaI. Folia subtus hirtella ...................forma hirtella NAKAI. Folia subtus incana ..................forma hypoleuca NAKAI.

Capitula parva oblonga cca. $1 \mathrm{~mm}$. lata. Folia pinnata, lacinis foliorum superiorum angustis $1-2 \mathrm{~mm}$. latis. Caulis elatus 3-8 pedalis. Panicula elongato-fusiformis. Squamæ 13. involucri fuscentes. ..........................Art. lavandulæfolia DC. 
Capitula $1.5-2.5 \mathrm{~mm}$. lata ovata v. oblongo-ovata v. obloga. Squamæ involucri scariaso-viridescentes v. albescentes. Mores plantæ variabillimi...............Art. vulgaris L.

73) Artemisia stelleriana BEss. var. sachalinensis NAKAI. A. stelleriana Regel Gart. Fl. (1886) p. 36. t. 498. Herder P1. Radd. III. iii p. 82. pro parte.

Habitat in Sachalin.

A typo differt, caule elatiore, racemo paniculato, capitulis minoribus. Insuper folia supra minus incana.

74) Artemisia lavandulæfolia DC. Prodr. VI. p. 119. Kom. F1. Mansh. III. p. 678.

A. vulgaris v. lavandulæfolia Maxim. Mél. Biol. VIII. p. 538. Fran. et SAv. Enum. Pl. Jap. II. p. 404. in clave.

A. vulgaris forma lavandulæfolia PaLIB. Consp. Fl. Kor. I. p. 116.

A. minutiflora NaKaI in Tokyo Bot. Mag. XXV. p. 56 et F1. Kor. II. p. 30.

A. vulgaris v. indica Hayata Comp. Form. p. 24 et Enum. P1. Form. p. 207. pro omnino.

Nom. Jap. Hime-jomogi.

Hab. Nippon media et australis, Shikoku et Kiusiu.

Distr. China, Manshuria, Korea et Formosa.

75) Artemisia sacrorum Leden. Fl. Alt. IV. p. 72. et Fl. Ross. II. p. 578.

var. latiloba LEDEB. 1. c.

Hab. in Jeso et in Sachalin.

var. intermedia Ledeb. 1. c. Maxim. in Mél. Biol. IX. p. 529. Koм. F1. Mansh. III. 663

forma viridis Ledeb. 1. c. KoM. 1. c.

A. Messerschmidtiana o. viridis f. typica NakaI Fl. Kor. II. p. 31.

A. sacrorum Koidzumi in Journ. Sci. Coll. XXVII. art. 13. p. 119.

In Sachalin et in Jeso vulgaris. 
var. laciniæformis NAKAI.

Forma A. laciniatæ similis sed rhachis foliorum pectinata et etiam A. Gmelini similis sed planta est major, folia longe latiora et quorum lacini multo elongati.

Hab. in Jeso.

76) Artemisia vulgaris L. Sp. P1. p. 848.

var. vulgatissima Bess. Abr. p. 53.

Multæ formæ cum hac adhuc confusæ fuerunt, sed non crescit in Japonia. In Europa et in Sibiria vulgaris est. Specimen Savatiere quod in Herbario Tokyoense servatur, est sine dubio planta Europæ.

var. gilvescens (MIQ.).

Art. gilvescens Miq. Prol. Fl. Jap. p. 107. Maxim. in Mél.

Biol. VIII. p. 536 in nota. Fran. et Sav. Enum. Pl. Jap. I. p. 239 pro p. II. p. 403.

Planta robusta $2-3$ pedalis. Caulis superior incano-lanatus. Folia subtus incana, ambitu ovata ve petiolum alatum subito attenuata, pinnatim incisa, lacinis ovato-lanceolatis acuminatis. Capitula 2-2.5 mm. lata.

In Jeso a $\mathrm{K}$ Iто lecta est. In Horto Botanico Koishikawense sub nomine Ojomogi colitur.

var. latiloba Ledeb. Fl. A1t. IV. p. 83. et Fl. Ross. II. p. 586. DC. Prodr. IV. p. 113.

Nom. Jap. Jama-Jomogi.

Planta robusta et elata 4-7 pedalis. Caulis superior incanotomentellus. Folia subtus incana ambitu ovata v. oblongoovata, pinnatim incisa, lacinis lanceolatis v. oblongo-lanceolatis. Capitula 2-2.5 mm. lata.

In Nippon boreale, Jeso et in Sachalin est vulgaris, in Nippon media in regionibus montanis tantum crescit.

var. indica (Willd.) Maxim. in Mel, Biol. VIII. p. 536. Fran. et Say. Enum. Pl. Jap. I. p. 240.

Art. indica WILld. Sp. Pl. III. p. 1846. DC. Prodr. VI. p. 114.

Planta humilis v. elata. Caulis superior incanus. Folia subtus nivea, ambitu ovata, pinnatim incisa, lacinis lanceolatis 
v. lineari-oblongis, acutis v. mucronatis, Capitula $1.5-2 \mathrm{~mm}$. lata.

forma $\alpha$. typica Nakai. Inflorescentia foliacea.

In Japonia tantum in Formosa crescere videtur.

forma $\beta$. nipponica Nakai. Inflorescentia non foliacea. Folia 3-6 cm. longa, lacinis oblongis v. lanceolatis v. angustis.

Nom. Jap. Jomogi.

In plano Nippon, Shikoku et Kiusiu nec non insulae Tsushima vulgaris.

forma montana NaraI. Folia usque $15 \mathrm{~cm}$. longa. Planta elata et robusta.

Nom Jap Ojomogiv Numa-jomogi.

In montibus Nippon media vulgaris.

var. Maximowiczii NaKaI.

A. vulgaris v. parviflora (non Bess.) Maxim. Prim. Fl. Amur. p. 160. Fran. et SAv. Enum. Pl. Jap. I. p. 252. II. p. 404. in clave.

Planta erecta $1-3$ pedalis. Caulis superior incanus. Folia subtus incana, ambitu ovata, inciso-pinnatifida, lacinis angustis 2-6 mm. latis. Capitula parva $1.5-2 \mathrm{~mm}$. lata.

in Nippon in plano cum Jomogi mixte crescit.

var. kamtschatica Bess. Tent. Abr. 54. DC. Prodr. VI. p. 113. Fr. SchmidT Fl. Sachal. p. 149.

A. vulgaris v. communis KoInz. in Journ. Coll. Sci. XXVII. art. 13. p. 120 p. p.

Planta erecta robusta. Caulis superior incana. Folia subtus incana, ambitu late-ovata, bipinnatifida, lacinis angustis. Capitula $2-2.5 \mathrm{~mm}$. lata.

Habitat in Sachalin, Jeso et in Kuril.

var. nipponica NAKAI. (varietas proxima ad varietatem mongolicam). Planta flexuosa, superior incana v. demum glabrescens. Folia subtus nivea, pinnatim v. subbipinnatim secta, lacinis angustis acuminatis $5-8 \mathrm{~mm}$. latis.

In silvis Nippon mediæ crescit.

Sect. III. Absinthium DC. F1. Fran. IV. p. 189. et Prodr. VI. p. 120. Ledeb. Fl. Ross. II. p. 594.

Receptaculum pilosum. Capitula heterogama. Flores radii feminæi, disci hermaphroditi. 
1. Folia sericea v. argenteo-sericea ..............................

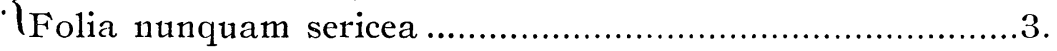

Folia omnia petiolata argenteo-sericea, bipinnata, lacinis 2. angustis $1 \mathrm{~mm}$. latis .................Art. Schmidtiana Maxim. Folia vulgo subsessilia v. sessilia, sericea, lacinis linearibus $1 \mathrm{~mm}$. latis Art. sericea WEB. Caulis 1-2 pedalis robustus. Folia glabrescentia v. glaberrima tripinnata, lacinis angustis $1 \mathrm{~mm}$. latis. Racemus subpaniculatus. Art. sinanensis YABE. $3 .\{$

Caulis $0.7-1$ pedalis. Folia sericeo-pubescentia. v. glabrescentia, tripinnatifida, lacinis lanceolatis $1.5-2 \mathrm{~mm}$. latis. Racemus simplex. ...........................Art. norvegica FrIEs. チシマモギ

(Continuatio sequitur). 